\section{0-12 RESEARCHING AN INNOVATIVE COTTAGE HOSPICE MODEL OF CARE: AN ACADEMIC AND HOSPICE PARTNERSHIP}

${ }^{1}$ Sean Hughes, ${ }^{1}$ Helen Barnes, ${ }^{2}$ Jo Yardley, ${ }^{1,2}$ Mary Turner, ${ }^{1,2}$ Catherine Walshe. ${ }^{1}$ International Observatory on End of Life Care, Lancaster University, UK; ${ }^{2}$ Hospice in the Weald, Tunbridge Wells, UK

\subsection{6/bmjspcare-2017-hospice.12}

Background Exploring innovative models of hospice, palliative and end of life care is imperative to meet contemporary demands in caring for those approaching the end of life. To this end, a new Cottage Hospice model is being developed in the south of England. The hospice concerned commissioned a concurrent two-year research study to track the programme development. This addresses the call for hospices to work with academics in determining which types of care work best (Payne, Preston, Turner, \& Rolls, 2013).

Aim To evaluate the development of Cottage Hospice using a research approach where solutions to challenges are developed collaboratively with a range of stakeholders. Results from this work will assist decision making as the programme proceeds. Methods Participatory Action Research is being used to evaluate the programme. A situational analysis using documents $(n=77)$ and interviews $(n=25)$ explored the programmes foundations. Action cycles $(n=4-8)$ in which issues are worked on in small groups to reach agreed solutions followed the initial phase. Findings will be fed back to stakeholders in workshops to share and refine results.

Results Initial results demonstrate a need for conceptual clarity about the model to be sought between staff, volunteers and service users in order to achieve a shared vision and support for the programme. Action cycles to address conceptual understandings and practical issues including staffing and family caregiver role are underway. It is anticipated that these will highlight areas for further action cycles.

Conclusions The early involvement of an academic research team in evaluating a new model of hospice care represents an embedded and enlightened approach in which research is not an afterthought. We believe this strengthens the basis for this new initiative. Gaining an in-depth, evidence based understanding of how challenges were resolved in the implementation of Cottage Hospice may be of use to others in the sector planning similar initiatives.

\section{Parallel session 4: Sharing care: different ways of working}

\section{0-13 THE ROLE OF THE CONSULTANT RADIOGRAPHER IN PALLIATIVE RADIOTHERAPY}

Linda Bedford. Musgrove Park Hospital, Taunton, UK

\subsection{6/bmispcare-2017-hospice.13}

Introduction Palliative patients account for $25 \%$ of the radiotherapy department's workload, however, many of these patients are not end of life and may live for years with a cancer diagnosis. Historically, they had a long wait to see consultant clinical oncologists and receive radiotherapy for their symptoms (the most common of which is pain) (Jones et al., 2014).
Service improvement A service has been developed for rapid access to palliative radiotherapy for patients with bone and brain metastases, to improve the patient experience and access timely symptom control. This is a Consultant Radiographer-led service where patients can be seen, assessed for radiotherapy and consent, treatment and radiation prescriptions completed. This reduces waiting times for patients as they do not need to be seen in clinics by Consultant Oncologists. This includes an outreach service within the trust and local hospices, which ensures all patients requiring palliative radiotherapy receive timely assessment and treatment.

Aims To assess patients individually in terms of suitability for palliative radiotherapy. Promote awareness of palliative radiotherapy as a treatment option in managing metastatic cancer as a complex long term condition. Reduce length of stay and admissions. Holistic pain management - liaising with palliative care teams in the community (Farrell, Merkley, \& Ingar, 2013).

Results A streamlined, direct referral process has been developed demonstrating continuity of care whilst educating staff, patients, families and carers on the use of palliative radiotherapy in the treatment of metastatic cancer. Pain is managed in a timely manner and optimised before radiotherapy treatment allowing a reduction in pain flare side effects.

Conclusion This work has highlighted the complex nature of palliative radiotherapy in the management of cancer but enables patients to access timely symptom control. Furthermore, development of a palliative MDT group including radiographers, dosimetrists and clinical oncologists is in place to assess the efficacy of the service.

\section{0-14 HOW DO INPATIENT HOSPICE VOLUNTEERS VIEW TRAINING AS A MEANS OF PREPARING THEM FOR THEIR ROLE?}

${ }^{1,2}$ Antonia Dean, ${ }^{1,2}$ Susan Willis. ${ }^{1}$ The Hospice of St Francis, Berkhamsted, UK; ${ }^{2}$ Sheffield Hallam University, Sheffield, UK

\subsection{6/bmjspcare-2017-hospice. 14}

Background There is a paucity of research on the training needs of hospice volunteers, despite acknowledgement that volunteers are crucial to the continued delivery of palliative care (Radbruch et al., 2010; Goosensen et al., 2016). The Commission into The Future of Hospice Care (2012) has called for a shared, core curriculum for the training of hospice volunteers but recognises the potential for unnecessary 'professionalisation' of volunteers, diluting their unique contribution. The volunteer perspective on training is not welladdressed in the literature.

Aims This study explores the views of UK inpatient hospice volunteers regarding initial training for role-preparation.

Methods A qualitative paradigm was selected with the aim of illuminating the volunteer experience. Data was collected by the audio-recording of two focus groups, with a combined total of 17 participants purposively recruited from volunteers working in a hospice inpatient unit. Thematic analysis was applied to transcripts of focus groups.

Results Six themes emerged: a) role preparation b) common sense and life experience c) role challenges, d) benefits and boundaries of training, e) training content, and f) training delivery. Volunteers perceived initial training to be important in increasing confidence and set out several areas where they 
felt additional training would be valuable. These included greeting and interacting with relatives, communication skills, cultural competence and the symptoms and signs of advanced illness. There was some disagreement about how training contributed to professionalisation and the extent of what education should be provided with respect to role boundaries. Volunteers advocated a mixed-methods approach to training, with an emphasis on scenario-based teaching and on-the-job mentoring and support.

Conclusion This study suggests areas of training that inpatient hospice volunteers perceive as worthwhile. It will inform local practice, shaping the education and support of future volunteers, and add to the wider evidence-base surrounding the development of this crucial group within the hospice workforce.

\section{0-15 A SHARED CARE PATHWAY FOR PEOPLE WITH ADVANCED LIVER DISEASE (ALD); INNOVATION OR IDEALISTIC?}

Virginia Campbell, Sharon Quinn. St Luke's Hospice, Basildon, UK

\subsection{6/bmjspcare-2017-hospice.15}

Background People with ALD have complex end-of-life needs. Palliative care provision or clear national guidance is limited. An innovative, unique joint hepatology/specialist palliative care project exploring a parallel planning approach has been established between the hospice and secondary care, which will lead the learning for hospices around ALD and non-malignant conditions.

Aims To explore the impact of this shared care approach on patient and carer outcomes and experience.

Methods Patients were recruited by the hospice Specialist Nurse Practitioner (SNP) from hepatology hospital clinics. The SNP offered holistic needs assessment, opportunities to discuss advance care planning and access to supportive care at the hospice whilst patients remained under hepatology management. Patients were reassessed four to eight weekly using OACC measures and the Short Form Liver Disease Quality of Life Assessment. Carers were assessed using CSNAT. In addition to analysis of patient data, hospital admission, A and E and GP visit avoidance was evaluated to assess health economics and quality of life.

Results A total of 38 patients were registered. At baseline our sample $(n=36)$ had a mean I-POS of 27.4 (range 4-64) and a mean AKPS score of 68.3(range 50.90). At the four month assessment, the sample $(n=23)$ had a mean I-POS of 19.7 (range 1-42) and a mean AKPS score of 77.0 (range 50-90). Preliminary analysis suggests that patients receiving early hospice intervention have reduced symptom burden and improved performance status. This was further supported by focus group feedback which highlighted continuity of care and open communication as contributing to improved well-being.

Conclusions Final data will be presented at the conference demonstrating key learning points from this collaborative and innovative approach which will lead and inform future service provision across hospices. Early and timely introduction to hospice services is beneficial to this marginalised group and this model is replicable for people with other non-malignant conditions.

\section{0-16 IMPROVING END OF LIFE CARE FOR ADULTS WITH SEVERE MENTAL ILLNESS AND LIFE-LIMITING CONDITIONS}

1,2,3 Jed Jerwood, 'Jane Coad, 'Diane Phimister, ${ }^{1}$ Nikki Holliday. ${ }^{1}$ Coventry University, Coventry, UK; ${ }^{2}$ John Taylor Hospice, Birmingham, UK; ${ }^{3}$ Birmingham and Solihull Mental Health Trust, UK

\subsection{6/bmjspcare-2017-hospice. 16}

Background People with mental illness experience higher rates of many life-limiting conditions and die on average twenty years earlier than the general population. A literature review was carried out which revealed limited understanding of the end of life needs of this patient group. The role of clinical staff was highlighted as a key factor, yet their views were not reflected in the published research.

Aims The aim of this research was to conduct an exploratory study to develop further understanding of how to improve end of life care for people with severe mental illness through exploring the views and experiences of clinical staff working in both mental health and end of life services.

Methods Four focus groups with clinicians were conducted, using the CUbe method (Magee, Fielden, \& Moody, 2015), across a large Mental Health Trust and a hospice. 23 participants from a range of professional backgrounds attended and shared their experiences and perceptions of the barriers to delivering good end of life care to people with mental illnesses. The data was analysed using the Framework Method (Gale, Heath, Cameron, Rashid, \& Redwood, 2013) and themes and explanatory concepts were drawn.

Findings The findings of the study were clustered into five themes: Structure of The System, Presentation of the Patient, Confidence of the Clinician and the Problem of Partnership. Implications for practice were drawn from the themes which can be used to inform improvements to clinical practice.

Conclusions The barriers to providing good end of life care were described within the literature, which largely originated outside the UK. The findings of the analysis found many similarities and some new findings. Recommendations were made which include improvements to partnership working, involvement of patients, carers and clinical staff in improving care and the need to develop the confidence and knowledge of clinicial staff from mental health and end of life services.

\section{Parallel sessions 5}

\section{Reaching out: primary care, care homes and ambulance services}

\section{0-17 GPs' EXPERIENCES AND PERCEPTIONS OF A HOSPICE SERVICE: A QUALITATIVE EVALUATION}

Sarah Onions, Sarah Mitchell, Jeremy Dale. University of Warwick, Warwick, UK

10.1136/bmjspcare-2017-hospice. 17

Background Most palliative and end of life care (PEOLC) is provided in primary care with referral to specialist palliative care (SPC) services for patients with more complex problems. Collaboration between general practitioners (GPs) and hospices 